\title{
Optimal Bang-Bang Controls for a Two-Compartment Model in Cancer Chemotherapy ${ }^{1}$
}

\author{
U. LEDZEWICZ ${ }^{2}$ and H. SCHÄTTLER ${ }^{3}$ \\ Communicated by M. Simaan
}

\begin{abstract}
A class of mathematical models for cancer chemotherapy which have been described in the literature take the form of an optimal control problem over a finite horizon with control constraints and dynamics given by a bilinear system. In this paper, we analyze a twodimensional model in which the cell cycle is broken into two compartments. The cytostatic agent used as control to kill the cancer cells is active only in the second compartment where cell division occurs and the cumulative effect of the drug is used to model the negative effect of the treatment on healthy cells. It is shown that singular controls are not optimal for this model and the optimality properties of bang-bang controls are established. Specifically, transversality conditions at the switching surfaces are derived. In a nondegenerate setting, these conditions guarantee the local optimality of the flow if satisfied, while trajectories will be no longer optimal if they are violated.
\end{abstract}

Key Words. Optimal control, cancer chemotherapy, cell cycle, compartment models, method of characteristics, strong minima.

\section{Introduction}

The mathematical models for cancer chemotherapy have a long history in mathematical biology (for instance, Refs. 1-5). While these approaches

\footnotetext{
${ }^{1}$ This material is based upon work supported by the National Science Foundation under Grant DMS-9971747. The first author was supported in part by an SIUE Summer Research Fellowship 2001 and an SIUE Research Scholar Award.

${ }^{2}$ Professor, Department of Mathematics and Statistics, Southern Illinois University at Edwardsville, Edwardsville, Illinois.

${ }^{3}$ Associate Professor, Department of Systems Science and Mathematics, Washington University, St. Louis, Missouri.
} 
based on quantifying dynamics and objective have their critics in the medical community (e.g. Refs. 6-7), in past years there has been renewed interest in these models, due to better models, and to a refinement of the techniques used to analyze the problems. For example, Kirschner, Lenhart, and Serbin (Ref. 8) consider an optimal control problem for chemotherapy of HIV which accounts for latently and actively infected $C D 4^{+} T$-cells; Fister and Panetta (Ref. 9) analyze a mathematical model which takes into account bone marrow destruction. In this paper, we analyze a two-dimensional model considered earlier in numerous papers by Kimmel, Swierniak, Polanski, and Duda (e.g. Refs. 10-13). This model, as well as the model considered in Ref. 9 and most other papers on the optimal control of mathematical models for cancer chemotherapy, is based on cell-cycle kinetics and treats this cell cycle as the object of control (Refs. 2,11, 14). We describe briefly the model and some of the biology behind it.

The state variable is given by the number of cancer cells and the control variable is the drug dosage. The active ingredient in the drug is a cytostatic agent which kills cancer cells and healthy cells alike. The goal is to maximize the number of cancer cells which the agent kills, while keeping acceptable the toxicity to the normal tissues. The dynamics of the cell cycle (within limits indeed) allows for such a feature by having the agents active at different stages. The cell cycle is modeled in the form of compartments which describe the different cell phases or, in simplified models, combine phases of the cell cycle into clusters. Each cell passes through a sequence of phases from cell birth to cell division. The starting point is a first growth phase $G_{1}$, after which the cell enters a phase $S$ where DNA synthesis occurs. Then, a second growth phase $G_{2}$ takes place in which the cell prepares for mitosis or phase $M$. Here, cell division occurs. Each of the two daughter cells can either reenter phase $G_{1}$ or for some time may simply lie dormant in a separate phase $G_{0}$ until reentering $G_{1}$, thus starting the entire process all over again.

The simplest mathematical models which describe the optimal control of cancer chemotherapy treat the entire cell cycle as one compartment. For instance, in Ref. 15, Chapter 6.7, a solution to the classical Gompertz model is given. Solutions to these single compartment models are not very informative due to the oversimplified nature of the model; better, more precise models (i.e., multicompartment models) need to be considered. Of these, still the simplest and at the same time most natural ones are models which divide the cell cycle into two and three compartments, respectively. The purpose of this division is to model the effects of the drugs used in chemotherapy in a more comprehensive way. In the two-compartment model, the application of the killing agent is limited to the $G_{1} / M$ phase when the cell is most vulnerable. The three-compartment model considers an additional cytostatic 
agent, a so-called blocking agent, which slows down the development of cells in the synthesis phase $S$. In this paper, we consider only the two-compartment model, but our methods apply equally to the more complex threecompartment model and we will report on these results elsewhere (Ref. 16).

So far, an analytical approach to problems of optimal control for cancer chemotherapy was limited to application of the Pontryagin maximum principle (Ref. 17). Beyond that, the controls and trajectories resulting from the maximum principle were analyzed only numerically (Refs. 18-19). This analysis centered around both bang-bang and singular controls. Although a bang-bang control seemed to be the more natural choice as candidate for optimality, and although it was even observed numerically that a singular protocol actually give the worst performance (Ref. 11), the question of the optimality of a singular control was left open (Refs. 12-13). In our earlier work (Refs. 20-21), we pursued a further analysis of this problem focusing precisely on the optimality of a singular control. Using the LegendreClebsch condition (a high-order necessary condition for optimality of singular arcs), we showed that these arcs are indeed locally maximizing rather than locally minimizing, and thus eliminated the singular controls from the candidates for optimality.

This paper is a continuation of our previous work; consequently, the main emphasis is on a complete analysis of the local optimality properties of bang-bang controls and corresponding trajectories. These controls are natural candidates for optimality and are widely used in a medical treatment where a maximum dose of chemotherapy is given repeatedly with breaks in between. We develop simple and easily verifiable conditions which allow us to determine the local optimality of bang-bang controls in nondegenerate cases. Our results are based on a recent construction by Noble and Schättler (Ref. 22). This construction embeds a trajectory corresponding to a bangbang control into a field of extremals and, using the method of characteristics, establishes the local optimality if all the switching surfaces are crossed transversally by the combined flow. It this condition is violated, an argument based on the construction of an envelope due to Sussmann (Ref. 23) is used to show that the trajectories will no longer be optimal. A simple corollary of our results is that bang-bang controls with one switch provide a relative minimum. This result was obtained earlier for specific parameter values by Nowakowski (Ref. 24) based on his construction of a concourse of flight (Ref. 25).

\section{Two-Compartment Model}

We review briefly the model following the exposition in Ref. 11. Taking into account only the phase sensitivity of the drug, the cell cycle is broken 
into two compartments of which the first combines the first growth phase $G_{1}$ and the synthesis phase $S$, while the second contains the second growth phase $G_{2}$ and the mitosis phase $M$. Let $N_{i}(t), i=1,2$, denote the number of cancer cells in the $i$ th compartment at time $t$. The transit times of the cells through phases of the cell cycle vary, particularly in malignant cells. In the simplest models, an exponential distribution is used to model the transit times and the expected number of cells exiting the $i$ th compartment is given by $a_{i} N_{i}(t)$, where $a_{i}$ is the parameter of the exponential distribution related to the inverse of the transit time. Assuming for the moment that no external stimuli are present, the inflow of the second compartment equals the outflow of the first. Therefore, we have

$$
\dot{N}_{2}(t)=-a_{2} N_{2}(t)+a_{1} N_{1}(t) \text {. }
$$

Cell division is represented by a factor 2 in the equation which describes the flow from the second into the first compartment,

$$
\dot{N}_{1}(t)=-a_{1} N_{1}(t)+2 a_{2} N_{2}(t) .
$$

Hence, the unperturbed dynamics of the cell cycle, or the number of cells in a particular compartment, can be represented by a system of linear ordinary differential equations if there are no external stimuli present.

The drug treatment influences the cell cycle in many possible ways and, in this model, only the most fundamental aspect is considered, that of cell killing. It is assumed that the cytotoxic agent is specific to the $G_{2} / M$ phases. This makes sense from a biological standpoint for a couple of reasons. First, in the mitosis $M$, the cell becomes very thin and porous; hence, the cell is more vulnerable to an attack while there will be a minimal effect on the normal cells. Second, chemotherapy during mitosis will prevent the creation of daughter cells. In our model, the control $u$ represents the dose of the drug administered, with the value $u=0$ corresponding to no treatment and the value $u=1$ corresponding to a maximum dose. It is assumed that the dose stands in direct relation to the fraction of cells which are being killed in the $G_{2} / M$ phase. Therefore, only the fraction $(1-u) a_{2} N_{2}$ of cells reenters the phase $G_{1} / S$ and undergoes cell division. However, all cells $a_{2} N_{2}$ leave the compartment $G_{2} / M$. Thus, the controlled mathematical model becomes

$$
\begin{array}{ll}
\dot{N}_{1}=-a_{1} N_{1}+2(1-u) a_{2} N_{2}, & N_{1}(0)=N_{10}, \\
\dot{N}_{2}=a_{1} N_{1}-a_{2} N_{2}, & N_{2}(0)=N_{20},
\end{array}
$$

with all the initial conditions positive. The performance index or objective is chosen as

$$
J=r_{1} N_{1}(T)+r_{2} N_{2}(T)+\int_{0}^{T} u(t) d t \rightarrow \min .
$$


In the objective, the coefficients $r_{1}$ and $r_{2}$ are weights and the penalty term $r_{1} N_{1}(T)+r_{2} N_{2}(T)$ represents a weighted average of the total number of cancer cells at the end of an assumed fixed therapy interval $[0, T]$. The number of cancer cells which do not undergo cell division at time $t$ and are killed is given by $u(t) a_{2} N_{2}(t)$; i.e., $u(t)$ is proportional to the fraction of ineffective cell divisions. Since the drug kills healthy cells at a proportional rate, the control $u(t)$ is used also to model the negative effect of the drug on the normal tissue or its toxicity. Using an $L_{1}$-objective rather than an $L_{2}$-objective on the control avoids distortions due to the square which would put a smaller penalty on lower doses. Thus, the integral in the objective models the cumulative negative effects of the treatment.

\section{Mathematical Model and Maximum Principle}

The dynamics of the two-compartment model is described by a bilinear system (Ref. 26). If we set $N=\left(N_{1}, N_{2}\right)$ and $r=\left(r_{1}, r_{2}\right)$, then the general form of the dynamics is

$$
\dot{N}(t)=(A+u B) N(t), \quad N(0)=N_{0},
$$

where $A$ and $B$ are fixed $2 \times 2$ matrices given by

$$
A=\left[\begin{array}{cc}
-a_{1} & 2 a_{2} \\
a_{1} & -a_{2}
\end{array}\right], \quad B=\left[\begin{array}{cc}
0 & -2 a_{2} \\
0 & 0
\end{array}\right],
$$

and the objective is to minimize

$$
J(u)=r N(T)+\int_{0}^{T} u(t) d t,
$$

over all Lebesgue measurable functions $u$ which take values in $[0,1]$, subject to the dynamics (6) and given initial condition $N(0)$. Note that, for any admissible control, the norm of matrix $A+u B$ is bounded over the interval $[0, T]$, and thus the right-hand side of the differential equation (6) is linearly bounded. Hence, it follows from well-known results about ordinary differential equations that the corresponding trajectory [i.e., the solution to (6)] exists on all of $[0, T]$.

Only the states $N(t)$ for which each component is positive are meaningful. Let

$$
\mathbb{P}=\mathbb{R}_{+}^{2}=\left\{N \in \mathbb{R}^{2}: N_{i}>0, i=1,2\right\} .
$$

It is seen directly that the dynamics has the structure

$$
\dot{N}_{i}(t)=-\beta_{i i}(t) N_{i}(t)+\beta_{i j}(t) N_{j}(t), \quad i \neq j ;
$$


in Eq. (9), regardless of the admissible control used, the functions $\beta_{i j}, i \neq j$, are nonnegative and the diagonal elements $\beta_{i i}$ are strictly positive. This structure implies that the physically meaningful part of the state space is positively invariant for the control system; i.e., if each coordinate of $N\left(t_{0}\right)$ is positive, then all the coordinates of $N(t)$ remain positive for all times $t \geq t_{0}$. Therefore, it is not necessary to add this condition as an extra state space constraint.

Proposition 3.1. If $N\left(t_{0}\right) \in \mathbb{P}$, then $N(t) \in \mathbb{P}$ for all $t \geq t_{0}$.

Proof. Let

$$
\tau=\min \left\{t \geq t_{0}: N_{i}(t)=0, \text { for some index } i\right\}<\infty,
$$

and denote by $k$ the index for which the minimum is achieved. Then we have that, on $[0, \tau)$,

$$
\dot{N}_{k}(t)=-\beta_{k k}(t) N_{k}(t)+\alpha(t),
$$

where

$$
\beta_{k k}(t)>0, \quad \alpha(t)=\beta_{k j}(t) N_{j}(t) \geq 0 .
$$

Thus,

$$
N_{k}(\tau)=\exp \left(-\int_{t_{0}}^{\tau} \beta_{k k}(s) d s\right)\left[N_{k}\left(t_{0}\right)+\int_{t_{0}}^{\tau} \exp \left(-\int_{t_{0}}^{s} \beta_{k k}(r) d r\right) \alpha(s) d s\right] .
$$

But the right-hand side is positive. Thus, a contradiction arises.

The first-order necessary conditions for optimality are given by the Pontryagin maximum principle (Ref. 17): If $u_{*}$ is an optimal control with corresponding trajectory $N_{*}$, then there exist a constant $\lambda_{0} \geq 0$ and an absolutely continuous function $\lambda$, which we write as a row vector $\lambda:[0, T] \rightarrow\left(\mathbb{R}^{2}\right)^{*}$ satisfying the adjoint equation and the transversality condition,

$$
\dot{\lambda}=-\lambda(A+u B), \quad \lambda(T)=\lambda_{0} r,
$$

such that

$$
\left(\lambda_{0}, \lambda(t)\right) \neq(0,0), \quad \text { for all } t \in[0, T],
$$

and the following minimum condition is satisfied: the optimal control minimizes the Hamiltonian

$$
H=\lambda_{0} u+\lambda(A+u B) N
$$


over the control set $[0,1]$ along $\left(\lambda_{0}, \lambda(t), N_{*}(t)\right)$. The multiplier $\lambda_{0}$ cannot vanish for this model [otherwise, also $\lambda(T)=0$ and thus $\lambda(t) \equiv 0$, contradicting the nontriviality of the multipliers]. Therefore, without loss of generality, we can normalize $\lambda_{0}=1$.

The same structure of the equations which implies the positive invariance of $\mathbb{P}$ for the flow of trajectories gives also the negative invariance of the first quadrant in the dual space,

$$
\mathbb{P}^{*}=\left\{\lambda \in\left(\mathbb{R}^{2}\right)^{*}: \lambda_{i}>0, i=1,2\right\},
$$

for the adjoint flow (11); i.e., if $\lambda(T) \in \mathbb{P}^{*}$, then $\lambda(t) \in \mathbb{P}^{*}$ for all times $t \leq T$. Hence, since $N_{0} \in \mathbb{P}$ and $r \in \mathbb{P}^{*}$, we have the following proposition.

Proposition 3.2. All states $N_{i}$ and costates $\lambda_{i}$ are positive over $[0, T]$.

\section{Singular Controls}

The optimal controls $u_{*}$ must satisfy the minimum condition of the maximum principle, i.e.,

$$
(1+\lambda(t) B N(t)) u_{*}(t)=\min _{0 \leq u \leq 1}(1+\lambda(t) B N(t)) u .
$$

Thus, if we define the so-called switching function $\Phi$ by

$$
\Phi(t)=1+\lambda(t) B N(t)
$$

then the optimal controls are given as

$$
u_{*}(t)= \begin{cases}1, & \text { if } \Phi(t)<0, \\ 0, & \text { if } \Phi(t)>0 .\end{cases}
$$

The control is not determined a priori by the minimum condition at the times when $\Phi(t)=0$. However, if $\Phi(t)$ vanishes on an open interval, then all its derivatives must also vanish, and this may determine the control. Controls of this kind are called singular, while we refer to the constant controls as bang controls. Then, the optimal controls need to be synthesized from these candidates.

The structure of the optimal controls is determined by the switching function and its derivatives. For instance, if $\Phi(t)=0$, but $\dot{\Phi}(t) \neq 0$, then the control has a switch at time $t$. Therefore, in order to analyze the structure of the optimal controls, we need to analyze the switching function and its derivatives. The following lemma, which allows to calculate first-order and higher-order derivatives of the switching function simply by calculating the commutators of matrices, is verified by an elementary direct calculation. 
Lemma 4.1. Let $M$ be a constant matrix, and let $\Psi(t)=\lambda(t) M N(t)$, where $N$ is a solution to the system equation (6) for the control $u$ and $\lambda$ is a solution to the corresponding adjoint equation. Then,

$$
\dot{\Psi}(t)=\lambda(t)[A+u B, M] N(t),
$$

where $[A, M]$ denotes the commutator of the matrices $A$ and $M$, defined as

$$
[A, M]=M A-A M \text {. }
$$

Note that we have chosen the order in the commutator so that it is consistent with the Lie derivative of the linear vector fields

$$
f(N)=A N \text { and } g(N)=M N .
$$

Indeed,

$$
\begin{aligned}
{[f, g](N) } & =D g(N) f(N)-D f(N) g(N) \\
& =M A N-A M N \\
& =[A, M] N .
\end{aligned}
$$

Therefore, in particular, we get for the switching function that

$$
\begin{aligned}
\dot{\Phi}(t) & =\lambda(t)[A, B] N(t), \\
\ddot{\Phi}(t) & =\lambda(t)[A+u(t) B,[A, B]] N(t) .
\end{aligned}
$$

Direct calculations verify the following relations for the matrices of the twocompartment model:

$$
\begin{aligned}
& {[A, B]=2 a_{1} a_{2}\left[\begin{array}{rr}
-1 & 0 \\
0 & 1
\end{array}\right]+\left(a_{1}-a_{2}\right) B,} \\
& {[A,[A, B]]=\left(a_{1}-a_{2}\right)[A, B]+4 a_{1} a_{2} B+4 a_{1}^{2} a_{2}\left[\begin{array}{ll}
0 & 0 \\
1 & 0
\end{array}\right],} \\
& {[B,[A, B]]=-4 a_{1} a_{2} B .}
\end{aligned}
$$

If the control $u$ is singular on an open interval $I$, then we have that

$$
0 \equiv \Phi(t)=\dot{\Phi}(t)=\ddot{\Phi}(t) .
$$

Solving for $u$ in (20) gives

$$
u_{\text {sin }}(t)=-\frac{\lambda(t)[A,[A, B]] N(t)}{\lambda(t)[B,[A, B]] N(t)},
$$

provided $\lambda(t)[B,[A, B]] N(t)$ does not vanish. Note that both the numerator and the denominator are scalar quantities, since we write $\lambda$ as a row vector. 
Since

$$
\Phi(t)=1+\lambda(t) B N(t) \equiv 0,
$$

we have

$$
\lambda(t)[B,[A, B]] N(t)=-4 a_{1} a_{2} \lambda(t) B N(t)=4 a_{1} a_{2}>0 .
$$

Thus, a simple calculation determines the singular control as

$$
u_{\sin }(t)=1-a_{1} \lambda_{2}(t) N_{1}(t) .
$$

A necessary condition for the optimality of a singular arc is the so-called Legendre-Clebsch condition (see for example Ref. 27), that $\lambda(t)[B,[A$, $B]] N(t)$ must be nonpositive along an optimal singular arc, and this condition is violated here. Thus, all singular arcs are slow or maximize the objective. Hence, we have the following proposition.

Proposition 4.1. Singular controls are not optimal.

Consequently (although more complicated structures like, for example, chattering arcs, which would have an infinite number of switchings, cannot be excluded a priori), bang-bang controls with only a finite number of switchings become the prime candidates for optimality.

\section{Bang-Bang Controls}

In this section, we develop conditions under which a bang-bang trajectory indeed gives a relative minimum over a set of controls for which the corresponding trajectories lie in some neighborhood of the reference trajectory in the time-state space. We will do this by constructing a parametrized family of extremals in the sense defined in Refs. 22, 28 by integrating the dynamic equation and the adjoint equation backward from the terminal time $T$ with the terminal condition $x(T)=p, p \in \mathbb{P}$ being a free parameter. The terminal values for all the adjoint variables are identical and given by the row vector $r$ of weights for the coordinates of the terminal state $N(T)$. This follows directly from the transversality condition of the maximum principle [see (11)]. Note that positivity of the trajectories needs to be enforced once we integrate the trajectories backward from a free terminal point $p$. Denoting the corresponding minimizing control by $u(t, p), 0 \leq t \leq T$, the dynamic equation for the system and the adjoint equation are given by

$$
\begin{aligned}
& \dot{N}(t, p)=(A+u(t, p) B) N(t, p), \\
& \dot{\lambda}(t, p)=-\lambda(t, p)(A+u(t, p) B),
\end{aligned}
$$


with terminal values

$$
N(T, p)=p, \quad \lambda(T, p)=r
$$

For bang-bang controls, these are linear equations with constant matrices between the switching times, hence easily solved. The crucial step is to determine the control $u(t, p)$, i.e., the correct switching sequence depending on the terminal value of the trajectory. This will be done through the minimum condition of the maximum principle. Then, the issue becomes whether the flow map $\sigma$ of the trajectories,

$$
\begin{aligned}
& \sigma:[0, T] \times \mathbb{P} \rightarrow[0, T] \times \mathbb{P}, \\
& (t, p) \mapsto \sigma(t, p)=(t, N(t, p)),
\end{aligned}
$$

defines a field, i.e., whether the corresponding curves cover the image $\sigma([0, T] \times \mathbb{P})$ injectively. It is shown in Ref. 22 that this will be the case if the restrictions of the flow away from the switching surfaces are diffeomorphisms and if proper transversality conditions (which guarantee that the combined flows are locally injective) are satisfied at the switching surfaces. Under these conditions, a differentiable solution to the Hamilton-JacobiBellman equation can be constructed and these controls are optimal (see for example Ref. 29). Here, we carry out this construction for the 2-compartment model making proper simplifications using the special structure of the model.

Let $\left(N_{*}, u_{*}\right)$ be a reference extremal pair, where $u_{*}$ is a bang-bang control with switchings at times $t_{i}, i=1, \ldots, m, 0<t_{m}<\cdots<t_{1}<t_{0}=T$, and $N_{*}$ is the corresponding trajectory; denote the corresponding adjoint variable by $\lambda_{*}$. We assume that all the switchings are strict in the sense that the derivative of the switching function at $t_{i}$ does not vanish,

$$
\dot{\Phi}_{*}\left(t_{i}\right)=\lambda_{*}\left(t_{i}\right)[A, B] N_{*}\left(t_{i}\right) \neq 0 \text {. }
$$

We call such a triplet $\Gamma=\left(N_{*}, u_{*}, \lambda_{*}\right)$ a strictly bang-bang extremal lift. We will show first that this assumption guarantees that we can imbed $\Gamma$ into a smoothly varying family of strictly bang-bang extremal lifts with an identical number of switchings which are close to the switchings of $u_{*}$. Our assumption excludes more degenerate situations when the switching function would have a local minimum or maximum at some switching time $t_{i}$. In this case, as the terminal point is being varied, the number of switchings in the corresponding controls will change. While it is still possible to construct a local field if $\dot{\Phi}\left(t_{i}\right)=0$, and while additional assumptions on higherorder derivatives are made like $\ddot{\Phi}\left(t_{i}\right) \neq 0$, the structure of the associated fields 
will be different and a separate analysis is required for each possible scenario. Here, we restrict our construction to the least degenerate or most typical situation. For this model, our assumption rules out only few exceptional trajectories.

Let $p_{*}=N(T)$ and, for $p$ in a neighborhood $W$ of $p_{*}$, integrate Eqs. (28)-(29) backward while choosing the control $u=u(t, p)$ so as to maintain the minimum condition of the maximum principle. Thus, $u\left(t, p_{*}\right)$ is given by the reference control $u_{*}$ and $N\left(t, p_{*}\right)$ and $\lambda\left(t, p_{*}\right)$ are the reference trajectory and corresponding multiplier.

Lemma 5.1. Let $\Gamma=\left(N\left(\cdot, p_{*}\right), u\left(\cdot, p_{*}\right), \lambda\left(\cdot, p_{*}\right)\right)$ be a strictly bangbang extremal lift, and denote the switching times of the control $u\left(\cdot, p_{*}\right)$ by $t_{i}, i=1, \ldots, m$ and $0<t_{m}<\cdots<t_{1}<t_{0}=T$. Then, there exists a neighborhood $W$ of $p_{*}$ and continuously differentiable functions $\tau_{i}$ defined on $W, i=1, \ldots, m$, such that, for $p \in W$, the controls $u(\cdot, p)$ are bang-bang with switchings in the same order as the reference control at the times $0<\tau_{m}(p)<\cdots<\tau_{1}(p)<T$ and the corresponding triplets $\Gamma_{p}=$ $(N(\cdot, p), u(\cdot, p), \lambda(\cdot, p))$ for $p \in W$ are strictly extremal lifts.

Proof. Inductively, we define the controls, trajectories, and multipliers integrating backward from the terminal time. For all $p$ in some open neighborhood $W$ and for $t \leq T$, let $u=u(t, p)$ be given by the value of the reference control $u_{*}$ on the interval $\left(t_{1}, T\right]$, and define $N(t, p)$ and $\lambda(t, p)$ as the solutions of (28)-(29), i.e.,

$$
\begin{aligned}
& N(t, p)=\exp \left((t-T)\left(A+u_{*} B\right)\right) p, \\
& \lambda(t, p)=r \exp \left((T-t)\left(A+u_{*} B\right)\right) .
\end{aligned}
$$

In particular, $N$ and $\lambda$ are smooth (in fact, analytic) functions. Defining

$$
\Phi(t, p)=1+\lambda(t, p) B N(t, p),
$$

it follows from the implicit function theorem that the equation

$$
\Phi(t, p)=0
$$

can be solved uniquely for $t$ in a neighborhood of $\left(t_{1}, p_{*}\right)$ and the solution is given by a smooth function $\tau_{1}=\tau_{1}(p)$ in a neighborhood of $p_{*}$. By choosing $W$ small enough, we can ensure that $\Phi(t, p)$ has no additional zeroes on the set

$$
D_{1}=\left\{(t, p): \tau_{1}(p) \leq t \leq T, p \in W\right\}
$$

and that

$$
\dot{\Phi}\left(\tau_{1}(p), p\right) \neq 0, \quad \text { for } p \in W .
$$


Hence, $(N(\cdot, p), u(\cdot, p))$ is an extremal over $\left[\tau_{1}(p), T\right]$ with corresponding multiplier $\lambda(\cdot, p)$. Without loss of generality, let $W$ be such a neighborhood.

Now, iterate the construction inductively from switching surface to switching surface. Suppose that $\tau_{i}=\tau_{i}(p)$ is given and that $N\left(\tau_{i}(p), p\right)$ and $\lambda\left(\tau_{i}(p), p\right)$ are smooth functions on $W$. Define $u(t, p)$ by the value of the reference control $u_{*}$ on the interval $\left(t_{i+1}, t_{i}\right)$ and again integrate the system and adjoint equations backward from $\left\{(t, p): t=\tau_{i}(p)\right\}$ with boundary conditions given by $N\left(\tau_{i}(p), p\right)$ and $\lambda\left(\tau_{i}(p), p\right)$. Then, it follows from standard results on the differentiable dependence of the solutions to ordinary differential equations that $N$ and $\lambda$ are still smooth functions. [Note that, since there is a switch in the control, these newly constructed states and costates do not agree with the ones constructed earlier for $t>\tau_{i}(p)$. This, however, is irrelevant for the construction.] Consequently, the equation $\Phi(t, p)=0$ can be solved again uniquely for $t$ in a neighborhood of $\left(t_{i+1}, p_{*}\right)$ and the solution is given by a smooth function $\tau_{i+1}=\tau_{i+1}(p)$ in a neighborhood of $p_{*}$. As before, by choosing $W$ sufficiently small, the resulting switching function will have no additional zeroes on

$$
D_{i}=\left\{(t, p): \tau_{i+1}(p) \leq t \leq \tau_{i}(p), p \in W\right\}
$$

and

$$
\dot{\Phi}\left(\tau_{i+1}(p), p\right) \neq 0, \quad \text { for } p \in W .
$$

Hence, the triplets $\Gamma_{p}=(N(\cdot, p), u(\cdot, p), \lambda(\cdot, p))$ constructed in this way are strictly bang-bang extremal lifts for $p \in W$.

While the states and costates remain continuous at the switching surface, their partials are discontinuous because of the different controls. For later use, we need to update the $p$-partials at a switching surface. Let $t=\tau(p)$ parametrize a switching surface and denote by $u_{+}$and $u_{-}$the constant controls for $t>\tau(p)$ and $t<\tau(p)$, respectively. Also, let

$$
\Delta u=u_{+}-u_{-}
$$

denote the jump in the control. For $p \in W$, let $N^{+}(t, p)$ and $N^{-}(t, p)$ denote the solutions to the system equation with controls $u_{+}$and $u_{-}$respectively and initial condition

$$
N^{ \pm}(t, p)=N(t, p), \quad \text { for } t=\tau(p) .
$$

Hence, the trajectories of the system are given by $N^{+}(t, p)$ for $t \geq \tau(p)$ and by $N^{-}(t, p)$ for $t \leq \tau(p)$. Thus, $N^{+}$and $N^{-}$are differentiable functions which agree on the set

$$
S=\{(t, p): t=\tau(p)\} .
$$


It is an elementary argument to see (for instance, see Lemma 2.6 in Ref. 22) that there exists a continuous real-valued function $\kappa(t, p)$ defined near $S$ such that, for $(t, p) \in S$, we have

$$
\operatorname{grad} N^{-}(t, p)=\operatorname{grad} N^{+}(t, p)+\kappa(t, p)[1,-\nabla \tau(p)] .
$$

Looking at the partials in $t$, we have that

$$
\dot{N}^{-}(t, p)=\dot{N}^{+}(t, p)+\kappa(t, p),
$$

and thus it follows from the dynamics that

$$
\begin{aligned}
\kappa(t, p) & =\left(A+u_{-} B\right) N(t, p)-\left(A+u_{+} B\right) N(t, p) \\
& =-\Delta u B N(t, p),(t, p) \in S .
\end{aligned}
$$

Hence,

$$
\frac{\partial N^{-}}{\partial p}(\tau(p), p)=\frac{\partial N^{+}}{\partial p}(\tau(p), p)+\Delta u B N(\tau(p), p) \nabla \tau(p) .
$$

An analogous formula holds for the multipliers $\lambda^{ \pm}(t, p)$ defined correspondingly. Note that $\left(\partial N^{-} / \partial p\right)(\tau(p), p)$ is a rank 1 correction of $\left(\partial N^{+} / \partial p\right)(\tau(p), p)$. For our construction, we need the matrix $(\partial N / \partial p)(t, p)$ to remain invertible. The partial derivative $\partial N / \partial p$ is the solution of the variational equation for (28). But since this equation is linear, the variational equation is identical and we have, for $t \leq \tau(p)$, that

$$
\frac{\partial N}{\partial p}(t, p)=\exp \left(\left(A+u_{-} B\right)(t-\tau(p))\right) \cdot \frac{\partial N^{-}}{\partial p}(\tau(p), p) .
$$

Hence $(\partial N / \partial p)(t, p)$ will be invertible if $\left(\partial N^{-} / \partial p\right)(\tau(p), p)$ is. Assuming inductively that $\left(\partial N^{+} / \partial p\right)(\tau(p), p)$ is nonsingular, it is a well-known result from linear algebra (see for instance Ref. 30) that $\left(\partial N^{-} / \partial p\right)(\tau(p), p)$ is nonsingular if and only if

$$
1+\nabla \tau(p)\left[\frac{\partial N^{+}}{\partial p}(\tau(p), p)\right]^{-1} \Delta u B N(\tau(p), p) \neq 0 .
$$

In fact, the inverse is given by

$$
\begin{aligned}
& {\left[\frac{\partial N^{-}}{\partial p}(\tau(p), p)\right]^{-1}} \\
& =\left[\frac{\partial N^{+}}{\partial p}(\tau(p), p)\right]^{-1}\left\{I d-\frac{\Delta u B N(\tau(p), p) \nabla \tau(p)\left[\left(\partial N^{+} / \partial p\right)(\tau(p), p)\right]^{-1}}{1+\nabla \tau(p)\left[\left(\partial N^{+} / \partial p\right)(\tau(p), p)\right]^{-1} \Delta u B N(\tau(p), p)}\right\} .
\end{aligned}
$$

In Ref. 22, the following result has been proven for bang-bang controls for general systems. 
Theorem 5.1. Let $\Gamma=\left(N_{*}, u_{*}, \lambda_{*}\right)$ be a strictly bang-bang extremal lift, and denote the switching times of the control $u_{*}$ by $t_{i}, i=1$, $\ldots, m, 0<t_{m}<\cdots<t_{1}<t_{0}=T$. Let $p_{*}=N_{*}(T)$, and let $\tau_{i}=\tau_{i}(p), i=1$, $\ldots, m, 0<\tau_{m}(p)<\cdots<\tau_{1}(p)<T$, be parametrizations of the switching times defined on some neighborhood of $p_{*}$. For the $i$ th switching, let

$$
\Delta u_{i}=u\left(t_{i}+\right)-u\left(t_{i}-\right)
$$

denote the jump in the control. For every switching $i=1, \ldots, m$, if we have that

$$
1+\nabla \tau_{i}\left(p_{*}\right)\left[\frac{\partial N^{+}}{\partial p}\left(t_{i}, p_{*}\right)\right]^{-1} \Delta u_{i} B N_{*}\left(t_{i}\right)>0,
$$

then $u_{*}$ is a relative minimum. More precisely, there exists a neighborhood $W$ of $p_{*}$ such that the flow $\sigma:[0, T] \times W,(t, p) \mapsto(t, N(t, p))$ defines a field of extremals and $u_{*}$ is optimal relative to any other control whose corresponding trajectory lies in the image $R=\sigma([0, T] \times W)$.

Sketch of Proof. Since $(\partial N / \partial p)(T, p)=I d$, the matrix $(\partial N / \partial p)(t, p)$ is invertible for $\tau_{1}(p) \leq t \leq T$. It follows inductively from (40) that the matrix $(\partial N / \partial p)(t, p)$ is nonsingular on all intervals $\left[\tau_{i+1}(p), \tau_{i}(p)\right], i=1, \ldots, m-1$, and on $\left[0, \tau_{m}(p)\right]$. The specific sign in the transversality condition (42) guarantees that the combined flow crosses transversally the switching surfaces. This allows us to combine the separate bang-bang flows into one injective flow. Furthermore, using (42), it can be shown that the corresponding value function remains continuously differentiable at the switching surface. Then, the optimality of $u_{*}$ relative to $R$ follows from standard Hamilton-Jacobi theory. The details of the argument can be found in Refs. 22 or 31 .

For this result to be of practical use, we need an efficient way to calculate the quantities

$$
\nabla \tau_{i}\left(p_{*}\right)\left[\frac{\partial N^{+}}{\partial p}\left(t_{i}, p_{*}\right)\right]^{-1} .
$$

Such a procedure exists and it requires neither the computation of the partial derivatives with respect to $p$ nor matrix inversion. To simplify the notation, we drop the index $i$. It follows from the implicit function theorem that

$$
\nabla \tau(p)=[(\partial \Phi / \partial p)(\tau(p), p)] / \Phi(\tau(p), p) .
$$




\section{Differentiating}

$$
\Phi(t, p)=1+\lambda(t, p) B N(t, p),
$$

we get

$$
\begin{aligned}
\frac{\partial \Phi}{\partial p}(t, p) & =\lambda(t, p) B \frac{\partial N}{\partial p}(t, p)+\left[\frac{\partial \lambda}{\partial p}(t, p) B N(t, p)\right]^{T} \\
& =\left\{\lambda(t, p) B+N^{T}(t, p) B^{T} S(t, p)\right\} \frac{\partial N}{\partial p}(t, p),
\end{aligned}
$$

where we define

$$
S(t, p)=\left[\frac{\partial \lambda^{T}}{\partial p}(t, p)\right]\left[\frac{\partial N}{\partial p}(t, p)\right]^{-1} .
$$

It is shown in Refs. 22, 28 that the matrix $S$ is the Hessian matrix of the value function $V$ associated with this family of parametrized extremals, i.e.,

$$
S(t, p)=\frac{\partial^{2} V}{\partial N^{2}}(t, N(t, p)),
$$

and that in general this matrix can be calculated as solution to a well-known Riccati equation (Ref. 29). For the case of bang-bang controls, this equation reduces to a linear Lyapunov equation; for bilinear systems, it even has constant coefficients, namely,

$$
\dot{S}(t, p)+S(t, p)(A+u B)+(A+u B)^{T} S(t, p) \equiv 0 .
$$

Thus, for $t \in\left[\tau_{i+1}(p), \tau_{i}(p)\right]$, we have

$$
\begin{aligned}
S(t, p)= & \exp \left((A+u B)^{T}\left(\tau_{i}(p)-t\right)\right) S\left(\tau_{i}(p), p\right) \\
& \exp \left((A+u B)\left(\tau_{i}(p)-t\right)\right) .
\end{aligned}
$$

Hence, we obtain the lemma below.

Lemma 5.2. The following result holds:

$$
\begin{aligned}
& \nabla \tau(p)\left[\frac{\partial N^{+}}{\partial p}(\tau(p), p)\right]^{-1} \\
& =-[1 / \dot{\Phi}(\tau(p), p)]\left[\lambda(\tau(p), p) B+N^{T}(\tau(p), p) B^{T} S(\tau(p), p)\right] .
\end{aligned}
$$

In particular, no partial derivatives of the state or costate with respect to the parameter $p$ need to be calculated nor do we need to calculate the inverse. Everything is subsumed in the calculation of the matrix $S(t, p)$. 
However, since $S$ is defined in terms of the $p$-partials, it will no longer be continuous at the switching times and we need to use the correct update formulas. As above, let $S^{+}(t, p)$ and $S^{-}(t, p)$ denote the matrices $S$ when constructed with $N^{ \pm}$and $\lambda^{ \pm}$, respectively. Then, analogously to (38), we have that

$$
\begin{aligned}
S^{-}(\tau(p), p)= & {\left[\frac{\partial\left(\lambda^{-}\right)^{T}}{\partial p}(\tau(p), p)\right]\left[\frac{\partial N^{-}}{\partial p}(\tau(p), p)\right]^{-1} } \\
= & {\left[-\Delta u B^{T} \lambda^{T}(\tau(p), p) \nabla \tau(p)+\frac{\partial\left(\lambda^{+}\right)^{T}}{\partial p}(\tau(p), p)\right] } \\
& \times\left[\Delta u B N(\tau(p), p) \nabla \tau(p)+\frac{\partial N^{+}}{\partial p}(\tau(p), p)\right]^{-1} .
\end{aligned}
$$

To simplify the notation, let

$$
\begin{aligned}
G_{\tau}(p) & =-\nabla \tau(p)\left[\frac{\partial N^{+}}{\partial p}(\tau(p), p)\right]^{-1} \Delta u \\
& =[\Delta u / \dot{\Phi}(\tau(p), p)]\left[\lambda(\tau(p), p) B+N^{T}(\tau(p), p) B^{T} S^{+}(\tau(p), p)\right] .
\end{aligned}
$$

Then, we have (all functions are evaluated at $(\tau(p), p)$ )

$$
\begin{aligned}
S^{-} & =\left(B^{T} \lambda^{T} G_{\tau}+S^{+}\right) \frac{\partial N^{+}}{\partial p}\left[\left(-B N G_{\tau}+I d\right) \frac{\partial N^{+}}{\partial p}\right]^{-1} \\
& =\left(B^{T} \lambda^{T} G_{\tau}+S^{+}\right)\left(I d-B N G_{\tau}\right)^{-1} \\
& =\left(B^{T} \lambda^{T} G_{\tau}+S^{+}\right)\left[I d+\frac{B N G_{\tau}}{1-G_{\tau} B N}\right] .
\end{aligned}
$$

Note that (see Lemma 5.2)

$$
\begin{aligned}
& 1-G_{\tau}(p) B N(\tau(p), p) \\
& =1+\nabla \tau(p)\left[\frac{\partial N^{+}}{\partial p}(\tau(p), p)\right]^{-1} \Delta u B N(\tau(p), p)>0,
\end{aligned}
$$

so that this matrix is well-defined. Thus, we have the following update formula for $S(t, p)$ at the switching $t=\tau(p)$.

Lemma 5.3. Let

$G_{\tau}(p)=[\Delta u / \dot{\Phi}(\tau(p), p)]\left[\lambda(\tau(p), p) B+N^{T}(\tau(p), p) B^{T} S^{+}(\tau(p), p)\right]$. 
Then,

$$
\begin{aligned}
S^{-}(\tau(p), p)= & {\left[B^{T} \lambda^{T}(\tau(p), p) G_{\tau}(p)+S^{+}(\tau(p), p)\right] } \\
& \times\left[\operatorname{Id}+\left[B N(\tau(p), p) G_{\tau}(p)\right] /\left[1-G_{\tau}(p) B N(\tau(p), p)\right]\right] .
\end{aligned}
$$

The formulas developed so far are valid in general for bang-bang controls of arbitrary single-input bilinear systems. A few simplifications occur for the 2-compartment model of cancer chemotherapy and we summarize them in the next theorem.

Theorem 5.2. Let $\Gamma=\left(N_{*}, u_{*}, \lambda_{*}\right)$ be a strictly bang-bang extremal lift with associated switching function

$$
\Phi_{*}(t)=1+\lambda_{*}(t) B N_{*}(t) .
$$

Denote the switching times of the control $u_{*}$ by $t_{i}, i=1, \ldots, m$, $0<t_{m}<\cdots<t_{1}<t_{0}=T$, and let $u_{i}$ be the value of the control on the interval $\left(t_{i}, t_{i-1}\right)$. Set $S_{0}^{-}=0$ and, for $i=1, \ldots, m$, define

$$
\begin{aligned}
S_{i}^{+} & =\exp \left(\left(A+u_{i} B\right)^{T}\left(t_{i-1}-t_{i}\right)\right) S_{i-1}^{-} \exp \left(\left(A+u_{i} B\right)\left(t_{i-1}-t_{i}\right)\right), \\
G_{i} & =-\left[1 /\left|\dot{\Phi}_{*}\left(t_{i}\right)\right|\right]\left[\lambda_{*}\left(t_{i}\right) B+N_{*}^{T}\left(t_{i}\right) B^{T} S_{i}^{+}\right], \\
S_{i}^{-} & =\left[B^{T} \lambda_{*}^{T}\left(t_{i}\right) G_{i}+S_{i}^{+}\right]\left[I d+\frac{B N_{*}\left(t_{i}\right) G_{i}}{1-G_{i} B N_{*}\left(t_{i}\right)}\right] .
\end{aligned}
$$

For $i=2, \ldots, m$, if we have that

$$
\left|\dot{\Phi}_{*}\left(t_{i}\right)\right|+N_{*}^{T}\left(t_{i}\right) B^{T} S_{i}^{+} B N_{*}\left(t_{i}\right)>0
$$

then all the matrices $S_{i}^{-}, i=2, \ldots, m$, are well-defined and $u_{*}$ yields a relative minimum for the 2-compartment model. More precisely, there exists a neighborhood $W$ of $p_{*}=N_{*}(t)$ such that the flow $\sigma$ restricted to $[0, T] \times W$ defines a field of extremals and $u_{*}$ is optimal relative to any other control whose trajectory lies in the image $R=\sigma([0, T] \times W)$.

Proof. The theorem follows from Theorem 5.1 using special properties of the model. We need to verify that condition (42) is satisfied at every switching; i.e., for $i=1, \ldots, m$, we have

$$
\begin{aligned}
& 1+\nabla \tau_{i}\left(p_{*}\right)\left[\frac{\partial N^{+}}{\partial p}\left(t_{i}, p_{*}\right)\right]^{-1} \Delta u_{i} B N_{*}\left(t_{i}\right) \\
& =1-\left[\left(u_{i}-u_{i+1}\right) / \dot{\Phi}_{*}\left(t_{i}\right)\right]\left[\lambda_{*}\left(t_{i}\right) B+N_{*}^{T}\left(t_{i}\right) B^{T} S_{i}^{+}\right] B N_{*}\left(t_{i}\right)>0 .
\end{aligned}
$$

By the minimum condition of the maximum principle, we have always that

$$
u_{i}-u_{i+1}=-\operatorname{sign}\left(\dot{\Phi}_{*}\left(t_{i}\right)\right) \text {. }
$$


For example, if $u_{i}=1$ and $u_{i+1}=0$, then the switching function decreases at $t_{i}$. Furthermore, for our model, $B^{2}=0$. Therefore, we have

$$
\begin{aligned}
& 1+\nabla \tau_{i}\left(p_{*}\right)\left[\frac{\partial N^{+}}{\partial p}\left(t_{i}, p_{*}\right)\right]^{-1} \Delta u_{i} B N_{*}\left(t_{i}\right) \\
& =1+\left[1 /\left|\dot{\Phi}_{*}\left(t_{i}\right)\right|\right] N_{*}^{T}\left(t_{i}\right) B^{T} S_{i}^{+} B N_{*}\left(t_{i}\right),
\end{aligned}
$$

and thus, conditions (42) and (60) are equivalent. In particular, these conditions guarantee inductively that the matrices $S_{i}^{-}, i=1, \ldots, m$, are well defined. Finally, at the terminal time, we have $N(T, p)=p$ and $\lambda(T, p)=r$, giving $S^{-}(T, p) \equiv 0$. In particular, it follows that $S^{+}\left(\tau_{1}(p), p\right) \equiv 0$; therefore, the first transversality condition (60) will be satisfied always for $i=1$.

Corollary 5.1. See Also Ref. 24. Every bang-bang trajectory with at most one switch yields a relative minimum for the problem.

Remark 5.1. The conditions (60) ensure that the switching surfaces are crossed in the same direction by the two flows involved in the switching. They form a true requirement which in general need not be satisfied. As a case in point, notice that, since $S_{1}^{+}=0$ and $B^{2}=0$,

$$
S_{1}^{-}=-B^{T} \lambda_{*}^{T}\left(t_{i}\right) \lambda_{*}\left(t_{i}\right) B /\left|\dot{\Phi}_{*}\left(t_{i}\right)\right|
$$

is negative semidefinite (and so is then $S_{2}^{+}$), so that the second term in (60) is nonpositive for $i=2$. In general, due to the continued addition of rank 1 matrices at the switching times, the matrices $S_{i}^{+}$will have no definiteness properties.

If the transversality condition (60) is violated with a negative value, then indeed local optimality of the bang-bang trajectory ceases at this switching.

Theorem 5.3. With the notation of Theorem 5.2, assume that the transversality condition

$$
\left|\dot{\Phi}_{*}\left(t_{i}\right)\right|+N_{*}^{T}\left(t_{i}\right) B^{T} S_{i}^{+} B N_{*}\left(t_{i}\right)>0
$$

is satisfied for $i=2, \ldots, l-1$, but that

$$
\left|\dot{\Phi}_{*}\left(t_{l}\right)\right|+N_{*}^{T}\left(t_{l}\right) B^{T} S_{l}^{+} B N_{*}\left(t_{l}\right)<0 .
$$

Then, there exists a neighborhood $W$ of $p_{*}=N_{*}(t)$ such that the flow $\sigma$ restricted to $D_{l}=\left\{\left(t, p: t_{l}<t \leq T, p \in W\right\}\right.$ defines a field of extremals and $u_{*}$ is optimal relative to any other control whose trajectory lies in the image $R_{l}=\sigma\left(D_{l}\right)$, but $u_{*}$ is no longer optimal for initial times $t \leq t_{l}$. 
Proof. The first statement follows from Theorem 5.2. More precisely, there exists a neighborhood $W$ of $p_{*}$ such that the parametrized controls for $p \in W$ are optimal when compared with all other controls which have the property that their trajectories lie in $D_{l}$. No optimality statement can be made with respect to trajectories which do not lie entirely in $D_{l}$. The neighborhood $W$ which defines the local field needs to be chosen so that all triplets $\Gamma_{p}=(N(\cdot, p), u(\cdot, p), \lambda(\cdot, p))$, for $p \in W$, are strictly extremal lifts and such that all the flows corresponding to the controls $u_{i}$ and $u_{i+1}$ cross the switching surfaces $\mathscr{S}_{i}, i=1, \ldots, l-1$, transversally and in the same direction. It is shown in Theorem 2.12 in Ref. 22 that, under these conditions, a differentiable solution $V$ to the Hamilton-Jacobi-Bellman equation can be constructed on $R_{l}$ implying the optimality of the corresponding controls $u(\cdot, p)$. Therefore, each of these controls is a strong relative minimum in the sense of the calculus of variations.

We now show that the extremals of the field which start at points on the $l$ th switching surface $\mathscr{S}_{l}$ are no longer optimal. This follows by carrying out an envelope argument due to Sussmann (Ref. 23) that shows actually that the $l$ th switching surface consists of conjugate points where local optimality ceases. For our model, all the necessary calculations can be given explicitly.

Let $t_{*}$ be the time of the $l$ th switching for the reference trajectory. The geometric meaning of condition (66) is that the flows corresponding to the controls $u_{l}$ and $u_{l+1}$ cross the switching surface $\mathscr{S}_{l}$ transversally near $\left(t_{*}, N_{*}\left(t_{*}\right)\right)$, but in opposite directions (Ref. 22, Theorem 3.12). Geometrically this implies that the combined flow, rather than crossing the switching surface, reflects off it to the same side. Clearly, one of the controls $u_{l}$ and $u_{l+1}$ is 0 and the other is 1 . Since the admissible controls are convex combinations of these, it follows that, at every point on $\mathscr{S}_{l}$, there exists a unique control $\bar{u}$ such that the vector $(A+\bar{u} B) N$ is tangent to the switching surface $\mathscr{S}_{1}$. Hence, there exists a positive $\kappa>0$ and an admissible control $\bar{u}$ defined on $\left[t_{*}, t_{*}+\kappa\right]$ such that the $(A+\bar{u}(t) B) N$ is tangent to the switching surface $\mathscr{S}_{1}$ for $t_{*} \leq t \leq t_{*}+\kappa$. Therefore, the corresponding trajectory $\bar{N}(t)$ with initial condition $\bar{N}\left(t_{*}\right)=N_{*}\left(t_{*}\right)$ lies entirely in the switching surface $\mathscr{S}_{1}$. Note that, since the dynamic equation for $u=0$ and $u=1$, strictly points to opposite sides of $\mathscr{S}_{l}$, it follows that $\bar{u}(t)$ takes values in the open set $(0,1)$. Furthermore, given any $\epsilon \in[0, \kappa]$, there exists a unique parameter $q_{\epsilon} \in W$ such that

$$
\bar{N}\left(t_{*}+\epsilon\right)=N\left(t_{*}+\epsilon, q_{\epsilon}\right) .
$$

This follows, since the flow $\sigma_{1}$ corresponding to the control $u_{l}$ is a local diffeomorphism near $p_{*}$, because the transversality condition (65) is satisfied 
for $i=l-1$. For $0 \leq \epsilon \leq \kappa$, we now define a one-parameter family of admissible input-trajectory pairs by taking as control

$$
u_{\epsilon}(t)=\left\{\begin{array}{ll}
\bar{u}(t), & \text { if } t_{*} \leq t<t_{*}+\epsilon \\
u\left(t, q_{\epsilon}\right), & \text { if } t_{*}+\epsilon \leq t \leq T
\end{array} .\right.
$$

Therefore, the corresponding trajectory is given by

$$
N_{\epsilon}(t)=\left\{\begin{array}{ll}
\bar{N}(t), & \text { if } t_{*} \leq t<t_{*}+\epsilon, \\
N\left(t, q_{\epsilon}\right), & \text { if } t_{*}+\epsilon \leq t \leq T
\end{array} .\right.
$$

Note that, for $\epsilon=0$, we get the reference trajectory $N_{*}$. The corresponding cost is given by

$$
C_{\epsilon}=\int_{t_{*}}^{t_{*}+\epsilon} \bar{u}(s) d s+C\left(t_{*}+\epsilon, q_{\epsilon}\right)
$$

where

$$
C(t, p)=\int_{t}^{T} u(s, p) d s+r N(T, p)
$$

denotes the cost of the parametrized extremal with initial condition $N(t, p)$ at time $t$. We will show below that the switching surface $\mathscr{S}_{l}$ is an envelope in the sense of Ref. 23; actually, it follows that all these trajectories have the same cost, i.e.,

$$
C_{\epsilon}=C_{0}, \quad \text { for all } \epsilon \in[0, \kappa] .
$$

But the controls $u_{\epsilon}$ for $\epsilon>0$ cannot be optimal, since the first portion $\bar{u}$ would be a singular arc, which is not optimal by Proposition 4.1. Therefore, $C_{0}$ can no longer be optimal.

Therefore, it suffices to show that the derivative of $C_{\epsilon}$ in $\epsilon$ vanishes on $[0, \kappa]$. Note that $C_{\epsilon}$ is absolutely continuous and, for almost every $\epsilon \in[0, \kappa]$, we have that

$$
(d / d \epsilon) C_{\epsilon}=\bar{u}\left(t_{*}+\epsilon\right)-u\left(t_{*}+\epsilon, q_{\epsilon}\right)+\frac{\partial C}{\partial p}\left(t_{*}+\epsilon, q_{\epsilon}\right)(d / d \epsilon) q_{\epsilon} .
$$

It follows from the shadow price lemma (Lemma 2.4 in Ref. 22) that

$$
\frac{\partial C}{\partial p}\left(t_{*}+\epsilon, q_{\epsilon}\right)=\lambda\left(t_{*}+\epsilon, q_{\epsilon}\right) \frac{\partial N}{\partial p}\left(t_{*}+\epsilon, q_{\epsilon}\right),
$$

where the partial derivatives of $N$ with respect to $p$ are calculated from the right with control given by $u_{l}$. The points $q_{\epsilon}$ are defined as solutions to the 
equation

$$
N\left(t_{*}+\epsilon, q_{\epsilon}\right)=\bar{N}\left(t_{*}+\epsilon\right) .
$$

Therefore, in terms of the flow $\sigma_{l}$ corresponding to the control $u_{l}$, we have

$$
\left(t_{*}+\epsilon, q_{\epsilon}\right)=\sigma_{l}^{-1}\left(t_{*}+\epsilon, \bar{N}\left(t_{*}+\epsilon\right)\right)
$$

and thus,

$$
\begin{aligned}
{\left[\begin{array}{l}
1 \\
(d / d \epsilon) q_{\epsilon}
\end{array}\right] } & \left(D \sigma_{l}\left(t_{*}+\epsilon, q_{\epsilon}\right)\right)^{-1}\left[\begin{array}{l}
1 \\
(d / d \epsilon) \bar{N}\left(t_{*}+\epsilon\right)
\end{array}\right] \\
= & {\left[\begin{array}{ll}
1 & 0 \\
(\partial N / \partial t)\left(t_{*}+\epsilon, q_{\epsilon}\right) & (\partial N / \partial p)\left(t_{*}+\epsilon, q_{\epsilon}\right)
\end{array}\right]^{-1}\left[\begin{array}{ll}
1 \\
(d / d \epsilon) \bar{N}\left(t_{*}+\epsilon\right)
\end{array}\right] } \\
= & {\left[\begin{array}{ll}
1 & 0 \\
\left.-[\partial N / \partial p)\left(t_{*}+\epsilon, q_{\epsilon}\right)\right]^{-1}\left[(\partial N / \partial t)\left(t_{*}+\epsilon, q_{\epsilon}\right)\right] & {\left[(\partial N / \partial p)\left(t_{*}+\epsilon, q_{\epsilon}\right)\right]^{-1}}
\end{array}\right] } \\
& \times\left[\begin{array}{l}
1 \\
(d / d \epsilon) \bar{N}\left(t_{*}+\epsilon\right)
\end{array}\right] .
\end{aligned}
$$

Hence,

$\left.(d / d \epsilon) q_{\epsilon}=\left[\frac{\partial N}{\partial p}\left(t_{*}+\epsilon, q_{\epsilon}\right)\right]^{-1}\left[(d / d \epsilon) \bar{N}\left(t_{*}+\epsilon\right)-(\partial N / \partial t)\left(t_{*}+\epsilon, q_{\epsilon}\right)\right)\right]$.

Since $\bar{N}$ is an actual trajectory of the system, we have almost everywhere

$$
(d / d \epsilon) \bar{N}\left(t_{*}+\epsilon\right)=\left(A+\bar{u}\left(t_{*}+\epsilon\right) B\right) \bar{N}\left(t_{*}+\epsilon\right),
$$

and from the parametrization of our extremals, it follows that

$$
(\partial N / \partial t)\left(t_{*}+\epsilon, q_{\epsilon}\right)=\left(A+u\left(t_{*}+\epsilon, q_{\epsilon}\right) B\right) N\left(t_{*}+\epsilon, q_{\epsilon}\right) .
$$

Therefore, since

$$
N\left(t_{*}+\epsilon, q_{\epsilon}\right)=\bar{N}\left(t_{*}+\epsilon\right),
$$

we obtain

$$
(d / d \epsilon) q_{\epsilon}=\left[\frac{\partial N}{\partial p}\left(t_{*}+\epsilon, q_{\epsilon}\right)\right]^{-1}\left[\bar{u}\left(t_{*}+\epsilon\right)-u\left(t_{*}+\epsilon, q_{\epsilon}\right)\right] B N\left(t_{*}+\epsilon, q_{\epsilon}\right)
$$


Hence,

$$
\begin{aligned}
(d / d \epsilon) C_{\epsilon} & =\left[\bar{u}\left(t_{*}+\epsilon\right)-u\left(t_{*}+\epsilon, q_{\epsilon}\right)\right]\left(1+\lambda\left(t_{*}+\epsilon, q_{\epsilon}\right) B N\left(t_{*}+\epsilon, q_{\epsilon}\right)\right) \\
& =\left[\bar{u}\left(t_{*}+\epsilon\right)-u\left(t_{*}+\epsilon, q_{\epsilon}\right)\right] \Phi\left(t_{*}+\epsilon, q_{\epsilon}\right)=0 .
\end{aligned}
$$

In the last line, recall that $\Phi$ denotes the switching function. Since $\left(t_{*}+\epsilon, q_{\epsilon}\right)$ parametrizes a point on the switching surface $\mathscr{S}_{l}$, this quantity vanishes. This completes the proof.

Figure 1 visualizes the geometric meaning of the transversality conditions (65) and (66). If the combined flow crosses the switching surfaces transversally like for the switching surface $\mathscr{S}_{l}$ [i.e., if condition (42) or (65) is satisfied], the trajectories cover the time-state space injectively and no local improvements are possible at such switching. But, if the flow reflects off the switching surface like for the switching surface $\mathscr{S}_{1}$ [i.e., condition (66) holds], then it is possible to do better even locally with exactly one switching less by eliminating the corresponding junction. In this case, there exist exactly two trajectories in our parametrization of bang-bang controls which start from points $q$ close to the switching surface $\mathscr{I}_{1}$. Of these, the one which ends at the terminal point $p$ and does not encounter $\mathscr{S}_{l}$ satisfies the sufficient conditions for optimality given in Theorem 5.2 and gives a strong local minimum. The trajectory which reflects off $\mathscr{S}_{l}$ and ends in $p^{\prime}$ is not optimal by Theorem 5.3. Intuitively, we can say that we can move down the flow to avoid the transversal fold. Note also that the locally optimal trajectory is a member of the field which has been constructed around the reference extremal. The switching surface $\mathscr{S}_{l}$ acts exactly like an envelope in the calculus of variations, and local optimality of the flow ceases there.

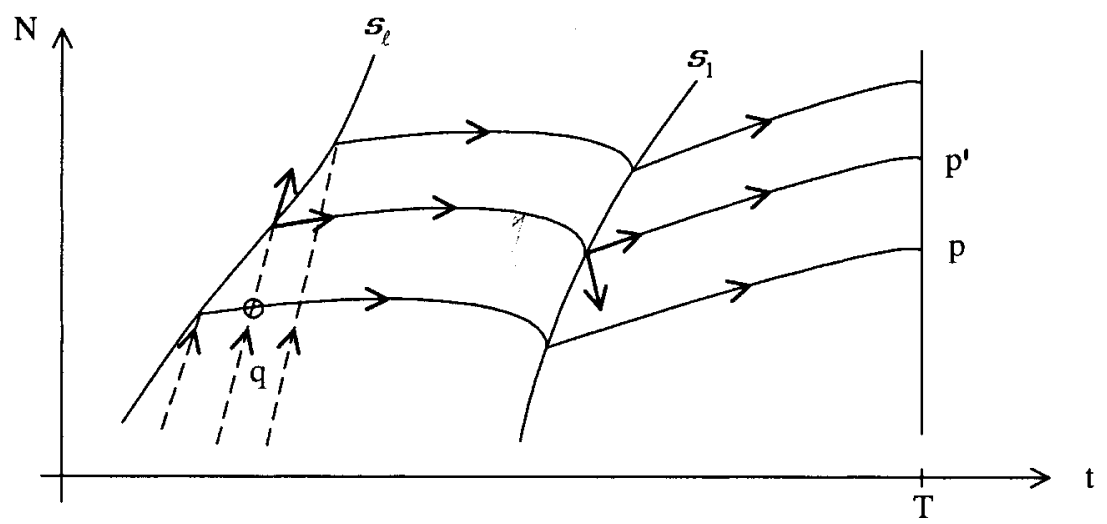

Fig. 1. Optimal and nonoptimal switchings. 
Figures 2 and 5 below show the graphs of two switching functions for our problem. The parameters chosen for the calculation were

$$
a_{1}=0.197, \quad a_{2}=0.356, \quad r_{1}=6.94, \quad r_{2}=3.94,
$$

the same values which have been used in the simulations in Ref. 11, and the time horizon is $T=10$. The trajectory with terminal conditions $p_{1}=p_{2}=0.3$ corresponding to the switching function in Fig. 2 has one switching and therefore is locally optimal by our results. However, the trajectory with terminal conditions $p_{1}=p_{2}=0.2$, violates the transversality condition at the second switching $t_{2}=1.2940$ [the value in the transversality condition (66) is given by -1.5086$]$, and thus only the portion over the interval $(1.2940,10]$ is locally optimal, but it is not optimal for its initial condition. Figures 3-4 and Figs. 6-7 show the corresponding trajectories verifying in particular that the states are positive. We have chosen these particular values of $p$ for mathematical reasons: as $p$ approaches the parameter values which correspond to a possible but not optimal singular arc (Ref. 21), the number of switchings increases, but these trajectories are no longer optimal.

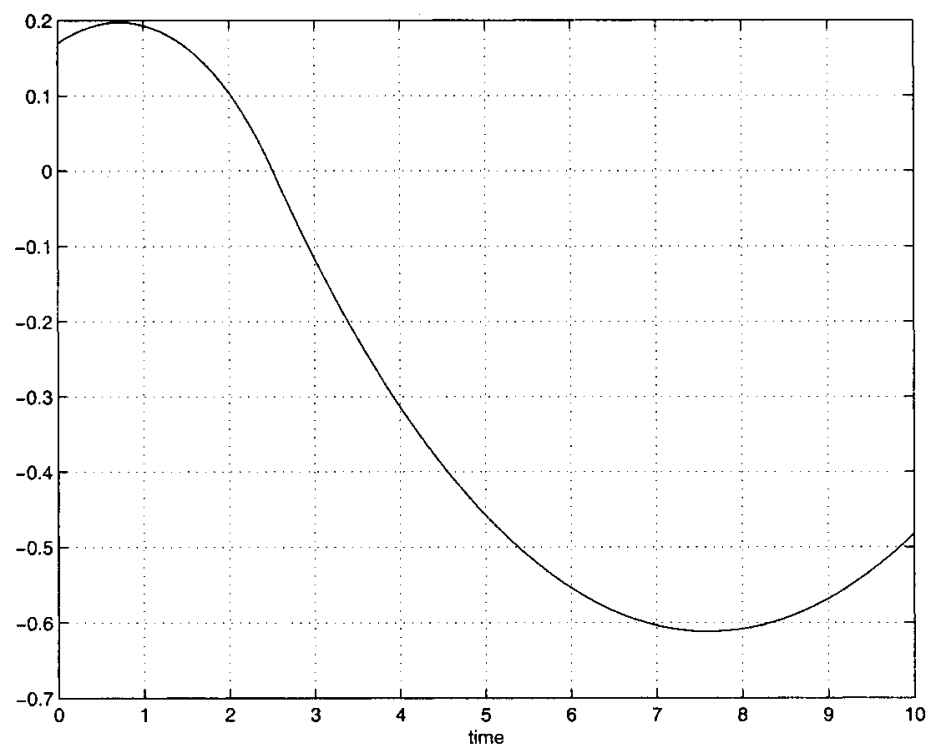

Fig. 2. Switching function $\Phi(t, p)$ for $p_{1}=p_{2}=0.3$. 


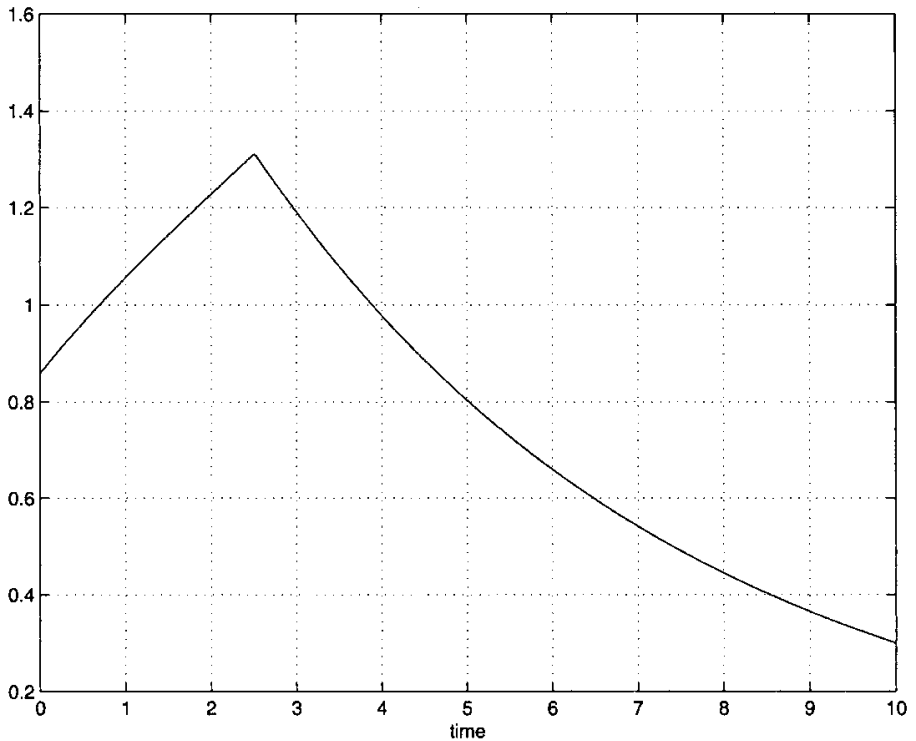

Fig. 3. $N_{1}$ for $p_{1}=p_{2}=0.3$.

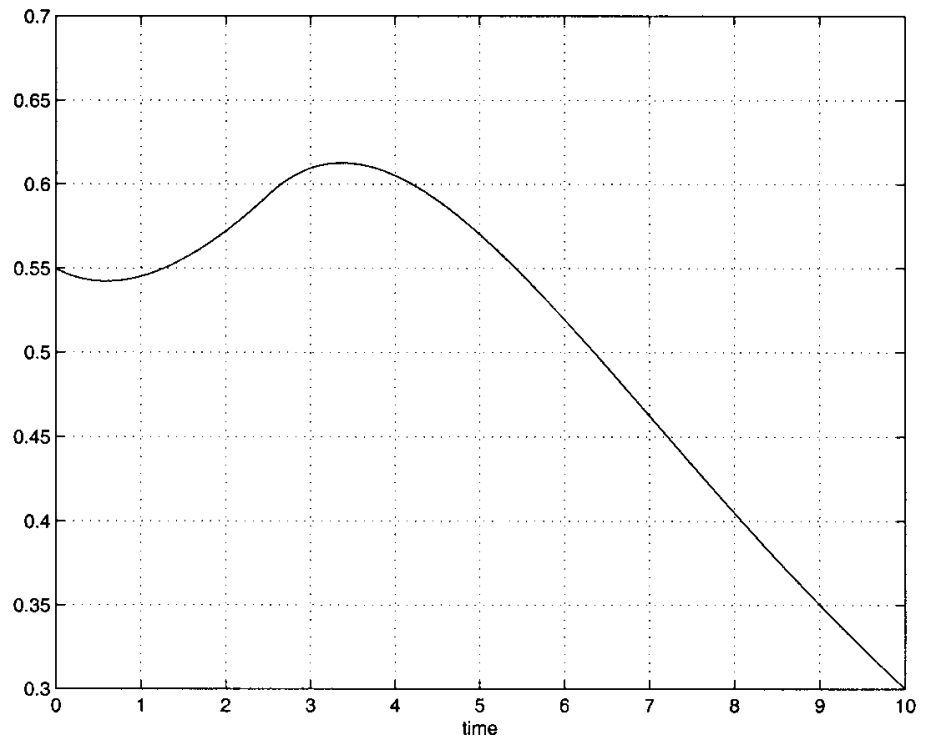

Fig. 4. $\quad N_{2}$ for $p_{1}=p_{2}=0.3$. 


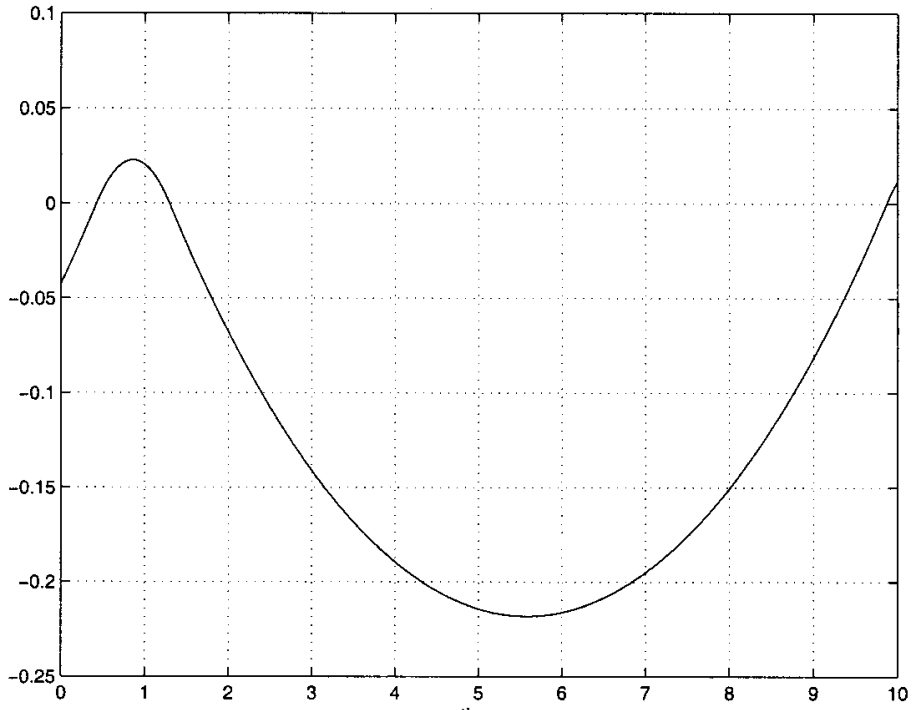

Fig. 5. Switching function $\Phi(t, p)$ for $p_{1}=p_{2}=0.2$.

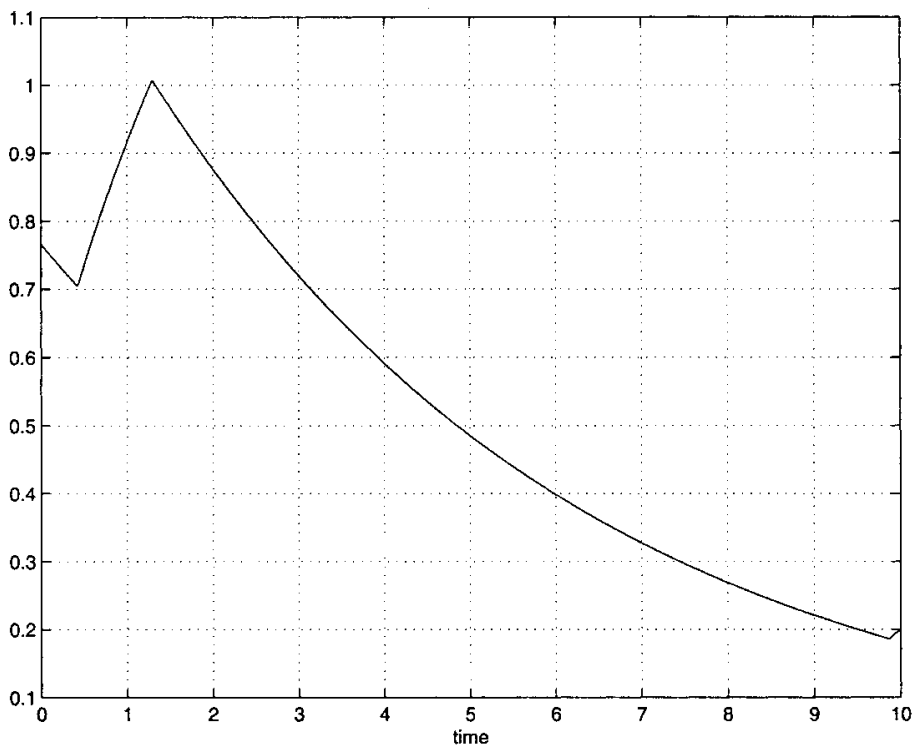

Fig. 6. $\quad N_{1}$ for $p_{1}=p_{2}=0.2$. 


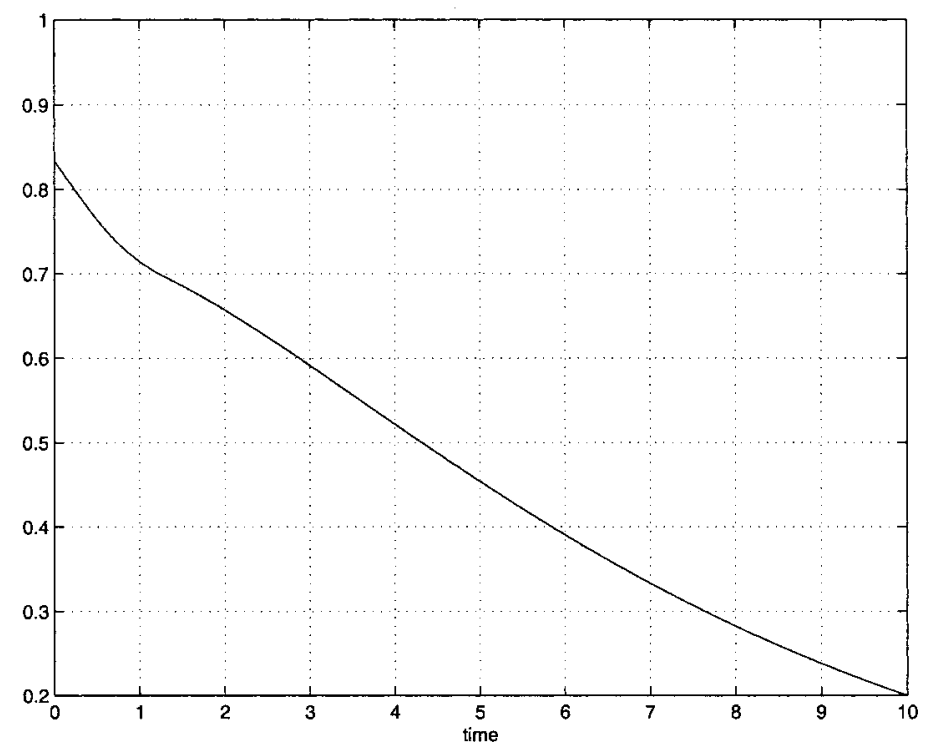

Fig. 7. $\quad N_{2}$ for $p_{1}=p_{2}=0.2$.

\section{Conclusions}

In the paper, we pursued a further analysis, beyond the standardly used maximum principle, of optimal controls of the 2-compartment model for cancer chemotherapy. Mathematically, with the use of the LegendreClebsch condition, singular controls were eliminated as candidates for optimality and an analysis of bang-bang controls was pursued. Using the method of characteristics, transversality conditions at the switching times were given [(42) and (66)] which characterize the geometric properties of the corresponding flow of trajectories. The results of our paper (Theorems 5.2 and 5.3) allow us to determine whether a given bang-bang control is at least locally optimal and to eliminate nonoptimal ones. Our constructions are local and the question about a global synthesis of the optimal controls still needs to be resolved.

From a biological point of view, eliminating singular controls from the candidates for optimality implies that it will not be optimal to administer the drug in chemotherapy at less than its maximum allowable dose. Bangbang controls, which then are left for analysis, correspond to protocols which alternate periods of administering the drug at a full dose with complete rest periods without chemotherapy in between. This coincides fully 
with current medical practice of scheduling full dose sessions with breaks in between. However, the number of sessions, their durations, and the timing of the rest periods have to be determined and some protocols, although they may satisfy the necessary conditions for optimality, may not be optimal (like the example given in the paper). With our transversality conditions [(42) and (66)], we can determine whether a proposed treatment protocol is optimal and cannot be improved. Following our constructions, a designed but nonoptimal protocol can be adjusted to be locally optimal by lengthening [respectively shortening, depending on the situation] the periods when the drug is being administered.

From a medical point of view, we believe that our analysis sheds some light into the design of chemotherapy protocols. Although we think the practitioner will find the model too simplified, our constructions are general and can be adapted easily to more complex systems; hence, they can be of use in the analysis of more complicated and more precise models.

\section{References}

1. EIsen, M., Mathematical Models in Cell Biology and Cancer Chemotherapy, Lecture Notes in Biomathematics, Springer Verlag, Berlin, Germany, Vol. 30, 1979.

2. Kim, M., Woo, K. B., and Perry, S., Quantitative Approach to the Design of Antitumor Drug Dosage Schedule via Cell Cycle Kinetics and Systems Theory, Annals of Biomedical Engineering, Vol. 5, pp. 12-33, 1977.

3. Martin, R. B., Optimal Control Drug Scheduling of Cancer Chemotherapy, Automatica, Vol. 28, pp. 1113-1123, 1992.

4. Swan, G. W., General Applications of Optimal Control Theory in Cancer Chemotherapy, IMA Journal of Mathematics and Applied Medical Biology, Vol. 5, pp. 303-316, 1988.

5. Swan, G. W., Role of Optimal Control in Cancer Chemotherapy, Mathematical Biosciences, Vol. 101, pp. 237-284, 1990.

6. TAnnock, I., Cell Kinetics and Chemotherapy: A Critical Review, Cancer Treatment Reports, Vol. 62, pp. xxx-xxx, 1978.

7. Wheldon, T. E., Mathematical Models in Cancer Research, Bristol Medical Science Series, Hilger, Town, State, 1988.

8. Kirschner, D., Lenhart, S., and Serbin, S., Optimal Control of Chemotherapy of HIV, Journal of Mathematical Biology, Vol. 35, pp. 775-792, 1997.

9. Fister, K. R., and Panetta, J. C., Optimal Control Applied to Cell-Cycle Specific Cancer Chemotherapy, SIAM Journal of Applied Mathematics, Vol. 60, pp. 1059-1072, 2000.

10. Kimmel, M., and Swierniak, A., An Optimal Control Problem Related to Leukemia Chemotherapy, Scientific Bulletin of the Silesian Technical University, Vol. 65, pp. 120-130, 1983. 
11. Swierniak, A., Polanski, A., and Kimmel, M., Optimal Control Problems Arising in Cell-Cycle Specific Cancer Chemotherapy, Cell Proliferation, Vol. 29, pp. 117-139, 1996.

12. Swierniak, A., and Duda, Z., Bilinear Models of Cancer Chemotherapy: Singularity of Optimal Solutions, Mathematical Population Dynamics, Vol. 2, pp. 347-358, 1995.

13. Swierniak, A., Polanski, A., and Duda, Z., Strange Phenomena in Simulation of Optimal Control Problems Arising in Cancer Chemotherapy, Proceedings of the 8th Prague Symposium on Computer Simulation in Biology, Ecology, and Medicine, pp. 58-62, 1992.

14. Swierniak, A., Cell Cycle as an Object of Control, Journal of Biological Systems, Vol. 3, pp. 41-54, 1995.

15. Roxin, E. O., Control Theory and Its Applications, Gordon and Breach Science Publishers, London, England, 1997.

16. Ledzewicz, U., and Schättler, H., Optimal Controls for a 3-Compartment Model of Cancer Chemotherapy, Journal of Biological Systems (to appear).

17. Pontryagin, L. S., Boltyanskit, V. G., Gamkrelidze, R. V., and MishCHenko, E. F., The Mathematical Theory of Optimal Processes, MacMillan, New York, NY, 1964.

18. Duda, Z., A Gradient Method for Application of Chemotherapy Models, Journal of Biological Systems, Vol. 3, pp. 3-11, 1995.

19. Duda, Z., Numerical Solutions to Bilinear Models Arising in Cancer Chemotherapy, Nonlinear World, Vol. 4, pp. 53-72, 1997.

20. Ledzewicz, U., and Schättler, H., Optimal Control for a Mathematical Model of Cancer Chemotherapy, Proceedings of the IASTED International Conference on Control and Applications, Edited by M. H. Hamza, IASTED/Acta Press, Anaheim, California, pp. 8-14, 2000.

21. Ledzewicz, U., and Schättler, H., On a Synthesis of Controls for a Mathematical Model of Cancer Chemotherapy, Proceedings of the 39th IEEE Conference on Decision and Control, Sydney, Australia, pp. 4845-4850, 2000.

22. Noble, J., and Schättler, H., Sufficient Conditions for Relative Minima of Broken Extremals in Optimal Control Theory, Journal of Mathematical Analysis and Applications (to appear).

23. Sussmann, H., Envelopes, High-Order Optimality Conditions, and Lie Brackets, Proceedings of the 28th IEEE Conference on Decision and Control, Tampa, Florida, pp. 1107-1112, 1989.

24. Nowakowski, A., The Proof That a Control with One Switch Is Optimal in a Simple Control Problem Arising in Chemotherapy, Proceedings of the Conference on Medical and Biological Applications of Mathematics, Warsaw, Poland, 1996.

25. Nowakowski, A., Field Theories in the Modern Calculus of Variations, Transactions of the American Mathematical Society, Vol. 309, pp. 725-752, 1988.

26. Mohler, R. R., Bilinear Control Systems, Academic Press, New York, NY, 1973.

27. Krener, A., The High-Order Maximal Principle and Its Application to Singular Controls, SIAM Journal on Control and Optimization, Vol. 15, pp. 256-293, 1977. 
28. Kiefer, M., and Schättler, H., Parametrized Families of Extremals and Singularities in Solutions to the Hamilton-Jacobi-Bellman Equation, SIAM Journal on Control and Optimization, Vol. 37, pp. 1346-1371, 1999.

29. Bryson, A. E., and Ho, Y. C., Applied Optimal Control, Hemisphere Publishing, Washington, DC, 1975.

30. Kailath, T., Linear Systems, Prentice Hall, Englewood Cliffs, New Jersey, 1980.

31. Noble, J., Parametrized Families of Broken Extremals and Sufficient Conditions for Relative Extrema, DSc Thesis, Department of Systems Science and Mathematics, Washington University, St. Louis, Missouri, 1999. 